\title{
CAMINA $p$-GROUPS THAT ARE GENERALIZED FROBENIUS COMPLEMENTS
}

\author{
by \\ I. M. Isaacs \\ Mathematics Department \\ University of Wisconsin \\ 480 Lincoln Dr. \\ Madison, WI 53706 \\ USA \\ E-mail: isaacs@math.wisc.edu \\ and \\ Mark L. Lewis \\ Department of Mathematical Sciences \\ Kent State University \\ Kent, OH 44242 \\ USA \\ E-mail: lewis@math.kent.edu
}

\begin{abstract}
Let $P$ be a Camina $p$-group that acts on a group $Q$ in such a way that $\mathbf{C}_{P}(x) \subseteq P^{\prime}$ for all nonidentity elements $x \in Q$. We show that $P$ must be isomorphic to the quaternion group $Q_{8}$. If $P$ has class 2, this is a known result, and this paper corrects a previously published erroneous proof of the general case.
\end{abstract}

Keywords: Camina group, Frobenius complement.

AMS Subject classification: Primary: 20D10, 20D15; Secondary 20C15. 
Let $G$ be a finite group that is neither perfect nor abelian, and recall that $G$ is said to be a Camina group if every nontrivial coset of $G^{\prime}$ is a conjugacy class of $G$, or equivalently, every nonlinear irreducible character of $G$ vanishes on $G-G^{\prime}$. (That these conditions really are equivalent is immediate from the fact that for $x \in G$, we have $\left|\mathbf{C}_{G}(x)\right|=\sum|\chi(x)|^{2}$, where the sum runs over $\chi \in \operatorname{Irr}(G)$.)

The purpose of this note is to prove the following, which appeared with an incorrect proof as Theorem 2 of [3].

THEOREM. Let $P$ be a Camina $p$-group, and suppose that $P$ acts on a nontrivial group $Q$ in such a way that $\mathbf{C}_{P}(x) \subseteq P^{\prime}$ for all nonidentity elements $x \in Q$. Then $P$ is the quaternion group of order 8 , and the action is Frobenius.

This theorem was used in [3] to give an alternative proof of a key step in the classification of Camina groups given in [1], where Dark and Scoppola proved that a Camina group must be either a $p$-group or a Frobenius group whose complement is either cyclic or $Q_{8}$. Unfortunately, as is explained in [3], the Dark-Scoppola proof ultimately relies on a flawed argument in [1]. Combining our result with Theorem 1 of [3], we now have what we hope is a correct (and simplified) proof of the Dark-Scoppola classification.

We begin with a fairly standard general result.

LEMMA. Let $A$ and $B$ be finite abelian groups, and suppose that there exists a nondegenerate bimultiplicative map $f: A \times B \rightarrow C$, where $C$ is a finite cyclic group. (This means that $f$ is a homomorphism in each variable and that the only elements $a \in A$ and $b \in B$ such that $f(a, B)=1$ or $f(A, b)=1$ are the identities of $A$ and $B$.) Then $A \cong B$.

Proof. Let $\mu$ be a faithful linear character of $C$. For each element $a \in A$, let $\lambda_{a}$ be the function on $B$ defined by $\lambda_{a}(b)=\mu(f(a, b))$. It is easy to check that the map $a \mapsto \lambda_{a}$ is an injective homomorphism from $A$ into the group $\widehat{B}$ of linear characters of $B$. Then $|A| \leq|\widehat{B}|=|B|$, and by symmetry, we have $|A|=|B|$. It follows that $A \cong \widehat{B} \cong B$, as required.

Next, we present a few easy results about Camina $p$-groups. Stronger versions of many of these are known, but they are scattered over a number of papers. (For example, see [1] and the references there.) Since the facts that we need can be established with elementary arguments, it seems reasonable to present the proofs here.

In the following, $P$ is always a Camina $p$-group.

PROPOSITION 1. $P / P^{\prime}$ is elementary abelian.

Proof. Let $x \in P$. If $x^{p} \notin P^{\prime}$, then $x \notin P^{\prime}$, so $\left|P: \mathbf{C}_{P}(x)\right|=\left|P^{\prime}\right|=\left|P: \mathbf{C}_{P}\left(x^{p}\right)\right|$, and thus $\left|\mathbf{C}_{P}(x)\right|=\left|\mathbf{C}_{P}\left(x^{p}\right)\right|$. Since $\mathbf{C}_{P}(x) \subseteq \mathbf{C}_{P}\left(x^{p}\right)$, we deduce that $\mathbf{C}_{P}(x)=\mathbf{C}_{P}\left(x^{p}\right)$. Now let $z \in \mathbf{Z}(P)$ have order $p$, and note that $z \in P^{\prime}$, so $x$ and $z x$ are conjugate. Then $x^{t}=z x$ for some element $t \in P$, and we have $\left(x^{p}\right)^{t}=\left(x^{t}\right)^{p}=(z x)^{p}=x^{p}$. It follows that $t \in \mathbf{C}_{P}\left(x^{p}\right)=\mathbf{C}_{P}(x)$, and this is a contradiction since $x^{t}=z x \neq x$. Thus $x^{p} \in P^{\prime}$, as required.

PROPOSITION 2. Suppose $P$ has nilpotence class 2. Then $\left|P: P^{\prime}\right|>\left|P^{\prime}\right|$. 
Proof. Let $x \in P-P^{\prime}$. Then $\left|P: \mathbf{C}_{P}(x)\right|=\left|P^{\prime}\right|$, so $\left|P: P^{\prime}\right|=\left|\mathbf{C}_{P}(x)\right|>\left|P^{\prime}\right|$, where the inequality is strict because $x \in \mathbf{C}_{P}(x)$.

¿From now on, we assume that $P$ has nilpotence class 3. (We mention that by the Theorem in [1], the nilpotence class of a Camina $p$-group cannot exceed 3 . We will use this fact in the proof of our main result.)

PROPOSITION 3. $\left[P^{\prime}, P\right]$ is elementary abelian.

In fact, more is true: $P^{\prime}$ is elementary abelian. (See the remarks preceding and following Proposition 6, below.)

Proof of Proposition 3 Since $\left[P^{\prime}, P\right]$ is abelian and is generated by elements of the form $[u, x]$, where $u \in P^{\prime}$ and $x \in P$, it suffices to show that $[u, x]^{p}=1$. Since $[u, x]$ is central in $P$, we have $[u, x]^{p}=\left[u, x^{p}\right]=1$, where the final equality holds since $P^{\prime}$ is abelian, and by Proposition 1, it contains both $u$ and $x^{p}$.

In the following, $Z=\mathbf{Z}(P)$ and $C=\mathbf{C}_{P}\left(P^{\prime}\right)$, and we note that $Z \subseteq P^{\prime} \subseteq C$.

PROPOSITION 4. Assume that $p>2$. Then $C / Z$ is elementary abelian.

Actually, it is not really necessary to assume that $p>2$ here or in Proposition 6 . This is because by Theorem 3.1 of [4], Camina 2-groups never have nilpotence class exceeding 2. (This fact too will be used in our proof of the main result.)

Proof of Proposition 4. Let $c \in C$ and $x \in P$, and write $c^{x}=c u$ and $u^{x}=u v$, where $u \in P^{\prime}$ and $v \in\left[P^{\prime}, P\right] \subseteq Z$. For positive integers $n$, it follows by induction that $c^{x^{n}}=c u^{n} v^{n(n-1) / 2}$. Since $p>2$ and $v^{p}=1$ by Proposition 3, we have $c^{x^{p}}=c u^{p}$. Also $x^{p} \in P^{\prime}$ by Proposition 1 , and thus since $c \in C=\mathbf{C}_{P}\left(P^{\prime}\right)$, we have $c^{x^{p}}=c$, and thus $u^{p}=1$. Now $\left(c^{p}\right)^{x}=\left(c^{x}\right)^{p}=(c u)^{p}=c^{p}$, where the last equality holds because $c$ and $u$ commute and $u^{p}=1$. Since $x \in P$ was arbitrary, it follows that $c^{p} \in Z$, and thus $C / Z$ has exponent $p$.

To see that $C / Z$ is abelian, note that $[P, C, C] \subseteq\left[P^{\prime}, C\right]=1$, so it follows by the three-subgroups lemma that $\left[C^{\prime}, P\right]=1$, and thus $C^{\prime} \subseteq Z$.

PROPOSITION 5. Assume that $Z$ is cyclic. Then $\left|C: P^{\prime}\right|$ is a square and $\left|C: P^{\prime}\right| \geq p^{2}$.

Proof. Since $Z$ is cyclic, $P$ has a faithful irreducible character $\chi$, and we argue that $\chi$ vanishes $P-Z$, and thus $|P: Z|=\chi(1)^{2}$. To see this, observe that $\chi$ vanishes on $P-P^{\prime}$ since $P$ is a Camina group, so it suffices to show that $\chi(x)=0$ for $x \in P^{\prime}-Z$. Since $x$ is noncentral, we can choose $t \in P$ with $x^{t}=x z$, where $z \in Z$ is some nonidentity element. Now $\chi_{Z}=\chi(1) \lambda$ for some faithful linear character $\lambda$ of $Z$, and thus $\chi(x)=\chi\left(x^{t}\right)=$ $\chi(x z)=\lambda(z) \chi(x)$. Since $\lambda(z) \neq 1$, it follows that $\chi(x)=0$, as wanted.

Now commutation defines a nondegenerate bimultiplicative map from $(P / C) \times\left(P^{\prime} / Z\right)$ into the cyclic group $Z$, and thus $|P: C|=\left|P^{\prime}: Z\right|$ by the lemma. Since $|P: Z|$ is a square, it follows that $\left|C: P^{\prime}\right|$ is a square. Also, $P / Z$ is a class 2 Camina group, so by Proposition 2, we have $\left|P: P^{\prime}\right|>\left|P^{\prime}: Z\right|=|P: C|$, and thus $C>P^{\prime}$, and we have $\left|C: P^{\prime}\right| \geq p^{2}$. 
PROPOSITION 6. Assume that $Z$ is cyclic and $p>2$. Then $P^{\prime}$ is elementary abelian.

As we have remarked, the assumption that $p>2$ is redundant here. In fact, as we will explain following the proof, the assumption that $Z$ is cyclic is not really needed either.

Proof of Proposition 6. By Proposition 5, we can choose an element $c \in C-P^{\prime}$. Now let $u \in P^{\prime}$ be arbitrary, and choose an element $t \in P$ such that $c^{t}=u c$. Since $c^{p} \in Z$ by Proposition 4, we have $c^{p}=\left(c^{p}\right)^{t}=\left(c^{t}\right)^{p}=(u c)^{p}=u^{p} c^{p}$, where the last equality holds because $c$ and $u$ commute. Then $u^{p}=1$, as required.

To see why it is not really necessary to assume that $Z$ is cyclic in Proposition 6 , observe that it suffices to show for each character $\chi \in \operatorname{Irr}(G)$ that the group $(P / \operatorname{ker}(\chi))^{\prime}$ is elementary abelian. Now $P / \operatorname{ker}(\chi)$ has a cyclic center, and it is either abelian, a Camina group of class 2 or a Camina group of class 3 . If it is abelian, there is nothing to prove and if it has class 3 , its derived subgroup is elementary by Proposition 6 . Finally, is easy to see using Proposition 1 that class 2 Camina $p$-groups always have elementary abelian derived subgroups.

Proof of Theorem. We proceed by induction on $|P|$. Observe first that the hypothesis guarantees that $P$ centralizes no nonidentity element of $Q$, and thus $|Q| \equiv 1 \bmod p$, and hence $Q$ is a $p^{\prime}$-group. Since $P$ is a Camina $p$-group, its nilpotence class is at least 2 . If $P$ has class 2, the result follows by Lemma 3.1 of [2], so we can assume that the class of $P$ is at least 3. Since Camina 2-groups have nilpotence class 2 by Theorem 3.1 of [4], we deduce that $p>2$, and we work to obtain a contradiction. Also, by the Theorem in [1] Camina $p$-groups have class at most 3 , and hence the class of $P$ must be 3 , exactly.

Since we can replace $Q$ by a nontrivial $P$-invariant subgroup, we can assume that $Q$ has no proper nontrivial $P$-invariant subgroup. It follows that $Q$ is an elementary abelian $q$-group for some prime $q \neq p$, and thus we can view $Q$ as an irreducible $F[P]$-module, where $F$ is the field of order $q$.

Let $K$ is the centralizer of the action of $G$ in the endomorphism ring of $Q$. Then $K$ is a finite division ring, and hence by Wedderburn's theorem, $K$ is a field. We can view $Q$ as a vector space over $K$, and as such, $Q$ is an absolutely irreducible $K[P]$-module. (We stress that we have not changed $Q$; what has changed is our point of view.) Let $\chi$ be the irreducible $q$-Brauer character of $P$ corresponding to the absolutely irreducible $K[P]$-module $Q$, and observe that in fact, $\chi \in \operatorname{Irr}(G)$ since $q$ does not divide $|P|$.

We argue now that $\chi$ is faithful. Otherwise, there exists a nontrivial central subgroup $U \subseteq \operatorname{ker}(\chi)$, and hence $U$ acts trivially on $Q$. Also, $P / U$ is nonabelian since $P$ has class 3 , and it follows that $P / U$ is a Camina $p$-group, so we can apply the inductive hypothesis to the action of $P / U$ on $Q$. Then $P / U$ has order 8 , and this is a contradiction since we have established that $p>2$.

Next, we show that no element of $P$ outside of $P^{\prime}$ can have order $p$. To see this, suppose that $H \subseteq P$, where $|H|=p$ and $H \cap P^{\prime}=1$. Since $P$ is a Camina group, $\chi$ vanishes on the nonidentity elements of $H$, and hence $\chi_{H}$ has a principal constituent. The action of $H$ on $Q$ is completely reducible, however, and it follows that $H$ has nontrivial fixed points in $Q$, and hence by hypothesis, $H \subseteq P^{\prime}$, and this is a contradiction.

Let $C=\mathbf{C}_{G}\left(P^{\prime}\right)$ and $Z=\mathbf{Z}(P)$, and note that $Z$ is cyclic since $\chi$ is faithful. Also $Z \subseteq P^{\prime}$, and thus $|Z|=p$ by Proposition 6 . If $c \in C-P^{\prime}$, then $c^{p} \in Z$ by Proposition 4 , 
and since $c$ does not have order $p$, we deduce that $c^{p}$ is a generator of $Z$.

Now $C / P^{\prime}$ is elementary abelian by Proposition 1 , and it has order at least $p^{2}$ by Proposition 5, and thus we can choose two elements $b, c \in C$ that generate distinct subgroups of order $p$ modulo $P^{\prime}$. Then $b^{p}$ and $c^{p}$ are generators of $Z$, and we can replace $c$ by a suitable power and assume that in fact, $b^{p}=c^{-p}$. Now write $[b, c]=z$, so $z \in Z$ by Proposition 4. Then $(b c)^{p}=b^{p} c^{p} z^{p(p-1) / 2}=b^{p} c^{p}=1$ because $p>2$ and $z^{p}=1$. Since $b$ and $c$ generate different subgroups of order $p$ in $C / Z$, it follows that $b c \notin P^{\prime}$, and since $b c$ has order $p$, this is our final contradiction.

\section{REFERENCES}

1. R. Dark and C. M. Scoppola, On Camina group of prime power order, J. Algebra 181 (1996), 787-802.

2. I. M. Isaacs, Coprime group actions fixing all nonlinear irreducible characters, Canad. J. Math. 41 (1989), 68-82.

3. M. L. Lewis, Classifying Camina groups: a theorem of Dark and Scoppola, Rocky Mountain J. Math. 44 (2014), 591-597.

4. I. D. Macdonald, More on p-groups of Frobenius type, Israel J. Math. 56 (1986), 335344. 